\title{
Near-Infrared Organic Fluorescent Nanoparticles for Long-term Monitoring and Photodynamic Therapy of Cancer
}

\author{
Qi Xia',2, Zikang Chen ${ }^{1,2}$, Yuping Zhou ${ }^{1,2}$, Ruiyuan Liu ${ }^{1 凶}$ \\ 1. Biomaterials Research Center, School of Biomedical Engineering, Southern Medical University, Guangzhou 510515, P.R. China \\ 2. School of pharmaceutical Sciences, Southern Medical University, Guangzhou 510515, P.R. China \\ $\triangle$ Corresponding author: R. Liu, E-mail: ruiyliu@smu.edu.cn \\ (C) Ivyspring International Publisher. This is an open access article distributed under the terms of the Creative Commons Attribution (CC BY-NC) license \\ (https://creativecommons.org/licenses/by-nc/4.0/). See http://ivyspring.com/terms for full terms and conditions.
}

Received: 2019.02.11; Accepted: 2019.03.21; Published: 2019.04.02

\begin{abstract}
Photodynamic therapy (PDT), which utilizes reactive oxygen species to ablate tumor, has attracted much attention in recent years. Photosensitizers with near-infrared (NIR) fluorescence as well as efficient ROS generation ability have been used for precise diagnosis and simultaneous treatment of cancer. However, photosensitizers frequently suffer from low ROS generation ability and NIR fluorescence quenching in aqueous media due to the aggregation.

Methods: We prepare an effective AIE active NIR emissive photosensitizer containing rhodanine as electron acceptor and triphenylvinylthiophene as electron donor is prepared, and encapsulate the corresponding photosensitizer into Pluronic F127 to fabricate NIR organic fluorescent nanoparticles. We then evaluate the NIR fluorescence bioimaging and photodynamic therapy ability of TPVTR dots in vitro and in vivo.

Results: The yielded organic fluorescent nanoparticles exhibit effective ROS generation ability, bright NIR emission, high photostability, and good biocompatibility. Both in vitro and in vivo experiments confirm that NIR organic fluorescent nanoparticles demonstrate good performances in long-term tracing and photodynamic ablation of tumor.

Conclusion: In summary, the synthesized organic fluorescent nanoparticles, TPVTR dots, showed great potentials in long-term cell tracing and photodynamic therapy of tumor. Our study highlights the efficient strategy for developing promising near-infrared organic fluorescent nanoparticles in advancing the field of bioimaging and further image-guide clinical applications.
\end{abstract}

Key words: AIE, near-infrared, organic fluorescent nanoparticles, long-term monitoring, photodynamic therapy

\section{Introduction}

Photodynamic therapy (PDT) is a promising therapeutic technique for Barrett's esophagus, age-related macular degeneration, and cancer [1-3], which utilizes photosensitizers in presence of light with an appropriate wavelength to generate reactive oxygen species (ROS) via intersystem crossingmediated singlet-to-triplet transition and subsequent energy transfers to eliminate or destroy diseased tissue by necrosis and apoptosis [4-6]. Compared to chemotherapy, radiotherapy, and surgery, PDT possesses less invasive, precise spatiotemporal control, negligible drug resistance, and fewer side effects [7-9]. To date, several types of photosensitizers have been developed for achieving effective PDT [10-12], such as boron dipyrromethene [13,14], phthalocyanine[15,16], and porphyrin[17]. As near-infrared (NIR) fluorescent bioimaging has significant advantages in high spatial resolution, deep penetration depth, high sensitivity, and direct visualization [18-22], the combination of NIR 
fluorescent bioimaging and photodynamic therapy in single molecular should help to improve the efficient of detection and simultaneous treatment of disease $[23,24]$. However, due to the hydrophobicity of aromatic macrocyclic structures, photosensitizers trend to aggregate in aqueous media which leads to quench fluorescence and reduce the efficiency for ROS generation[25]. Moreover, the existence of fluorescent quenching effect also makes it difficult to develop organic fluorescent nanoparticles with bright NIR emission and efficient ROS production ability for image-guided PDT based on traditional photosensitizers. Hence, many strategies have been developed to improve the diagnosis and treatment efficiency, such as modification with hydrophilic groups or load in nanocarrier platform such as micelles [26,27], upconversion nanoparticles [28], metal nanodots [29], silica nanoparticles [28], and metal-organic frameworks [31,32]. Despite of the achieved effect of these studies, the development of novel photosensitizers with enhanced ROS production efficiency as well as NIR fluorescence in aggregated state is highly desirable.

Recently, organic fluorescent nanoparticles based on AIE active fluorogens, which exhibit bright fluorescence in aggregated state, have been investigated and shown great application prospects in cell tracking [33], tumor imaging [34,35], and imageguided treatment[36]. Opposite to conventional photosensitizers, AIE active fluorophores containing electron donor (D)-acceptor (A) structure exhibit efficient ROS production in aggregate state [37-39]. Meanwhile, the ideal AIE active fluorogens also show far red/NIR fluorescence with large Stokes shifts, which are highly desirable for optical bioimaging [40]. These unique characteristics enable a great potential for image-guided PDT by encapsulation of AIE active fluorophores by polymer matrix to provide platform with NIR fluorescence for bioimaging[3], ROS production for ablation [41], negligible dark toxicity [42], good photostability[41], and biocompatibility [44]. For example, NIR AIE active probe containing tetraphenyl ethene (TPE) as electron donor and dicyanovinyl (DC) as acceptor, TPETCAQ, is encapsulated by DSPE to yield organic fluorescent nanoparticles for image-guided PDT [41]. Hence, AIE active fluorogens containing D-A structure are promising candidate for fluorescent bioimaging as well as photodynamic therapy.

In this contribution, we designed and synthesized AIEgen with rhodanine as electron acceptor and tetraphenylethylene as electron donor, and enhanced D-A structure by introducing electron-rich groups of thiophene, making the emission wavelength red-shifted to near infrared region. The novel NIR fluorogen, TPVTR, shows distinctly AIE feature and ROS generation property in the aggregate state. The corresponding organic fluorescent nanoparticles were fabricated by encapsulation TPVTR within Pluronic F127, which exhibit high photostability and good biocompatibility, and demonstrate superior performances in photodynamic ablation and long-term monitoring of tumor in vitro and in vivo.

\section{Results and Discussion}

\section{Fabrication and Characterization of TPVTR dots}

The synthetic route toward TPVTR is presented in Scheme 1A. Suzuki coupling between bromotriphenylethylene and 5-formylthiophene-2-boronic acid yielded the precursor TPVT-CHO. Then, the Knoevenagel condensation reaction between TPVT$\mathrm{CHO}$ and rhodanine was carried out to afford the targeted product TPVTR. Detailed preparation process and characterization data are provided in the Experimental Section.

The fluorescent property of TPVTR in THF/water mixed solvents are shown in Figure 1A and Figure 1B. It can be seen that when the water percentage $\left(f_{\mathrm{w}}\right)$ is less than $60 \%$, the PL intensity is very weak and keeps stable. Upon further increase of the $f_{w}$, the fluorescent intensity of TPVTR significantly enhances due to the formation of aggregates. Due to the D-A structure of TPVTR, the further enhancement of $f_{w}$ will increase the TICT effect leading to the decrease of fluorescent intensity. Therefore, these results confirm that TPVTR is AIE active.

Due to the hyrophobic property of TPVTR, TPVTR was encapsulated in Pluronic F127 to fabricate TPVTR nanoparticles (TPVTR dots) via nanoprecipitation method. The size and morphology of TPVTR dots were recorded and observed by dynamic light scattering (DLS) and transmission electron microscopy (TEM). As shown in Figure 1C, TPVTR dots have good appearance and uniform particle size $(\sim 100 \mathrm{~nm})$. The particle size change of TPVTR dots which are stored in PBS at room temperature up to 15 days was monitored by DLS (Figure S5A), and there is no obvious aggregation and precipitation, indicating that TPVTR dots have excellent colloidal stability.

As shown in Figure 1D, the UV-vis absorption spectrum of TPVTR dots covers range of $400 \sim 600 \mathrm{~nm}$ with a maxmium around $485 \mathrm{~nm}$, the emission spectrum arranges from 600 to $900 \mathrm{~nm}$ with a peak around $695 \mathrm{~nm}$, belongs to the NIR region, which is beneficial to be efficient photosensitizer for PDT application. Interesting, TPVTR dots exhibited long Stokes shift which is about $210 \mathrm{~nm}$. To further verify the fluorescent stability of TPVTR dots, TPVTR dots 
were stored in PBS solutions with long-term or in PBS with different $\mathrm{pH}$ ranging from 3 to 12 . The results show that the fluorescent intensity of TPVTR dots in PBS solutions changes slightly after 15 days (Figure S5A). Meanwhile, the photoluminescence intensity of TPVTR dots keeps stable with the change of $\mathrm{pH}$ value (Figure S5B). All these results confirm that TPVTR dots have good stability, which is beneficial for subsequent applications in biological imaging.

The ROS generation efficiency of TPVTR dots in PBS solutions was subsequently evaluated under the visible-light $\left(400 \sim 750 \mathrm{~nm}, 200 \mathrm{~mW} \cdot \mathrm{cm}^{-2}\right)$ irradiation using 9,10-anthracenediylbis(methylene)dimalonic acid (ABDA) $(50 \mu \mathrm{M})$ as an indicator. As shown in Figure 1E, the decrease of ABDA absorption over a time period from $0 \mathrm{~s}$ to $270 \mathrm{~s}$ is observed. The absorbance of ABDA and TPVTR dots mixture decreases a lot $(72 \%)$ after irradiation for $270 \mathrm{~s}$, which means that ABDA is consumed by the ROS generated from TPVTR dots $(5 \mu \mathrm{M})$. As a control, the absorption of ABDA without TPVTR dots remains about $90 \%$ under irradiation of the same time, which confirms that the absorbance of ABDA under light irradiation is relatively stable. The intensity of $A / A_{0}$ in Figure $1 \mathrm{~F}$ also demonstrates that TPVTR dots can generate ROS effectively. All the results confirm that TPVTR dots exhibit the ability of generation ROS and can be

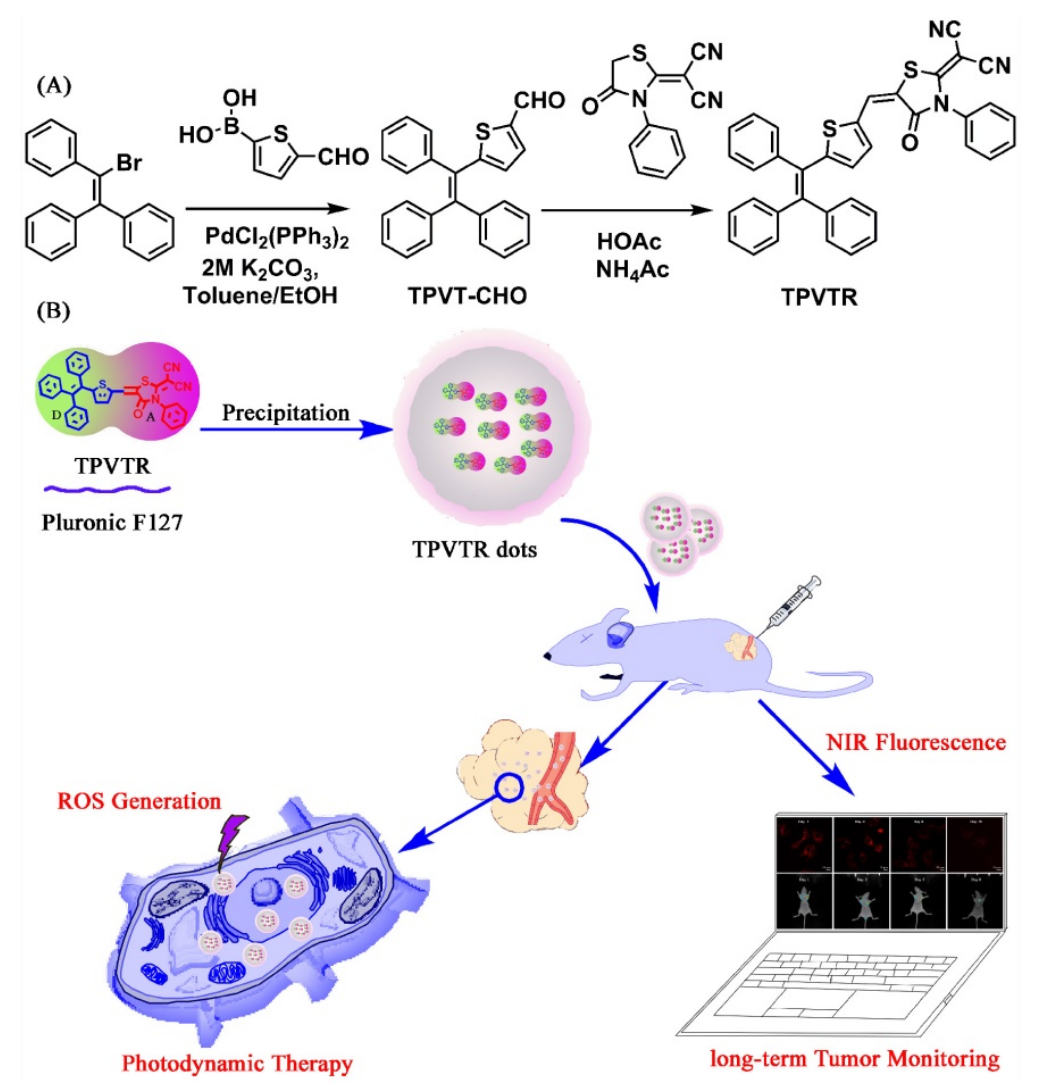

Scheme 1. (A) Synthetic routine to TPVTR. (B) Schematic illustration of the fabrication of TPVTR dots, long-Term Monitoring and Photodynamic Therapy of Cancer. potentially used as photodynamic therapy agent.

\section{In vitro Cell Tracing and In vivo Monitoring Living Tumor Growth}

With the advantages of NIR emission of TPVTR dots, the activation of TPVTR dots in cells was also studied by co-cultured TPVTR dots with HepG2 cells. As illustrated in Figure 2, TPVTR dots can penetrate into cells, accumulate in the cytoplasm and show bright NIR fluorescence. In addition, the photostability of TPVTR dots under continuous laser irradiation up to $10 \mathrm{~min}$ was also investigated. After HepG2 cells incubated with TPVTR dots were irradiated by $10 \mathrm{~min}$ continuous laser, the fluorescent intensity of TPVTR dots in cells exhibits less change than DAPI. These dates indicate that TPVTR dots could be a promising optical material for bioimaging applications.

Thanks to the good performance (e.g. high photostability, NIR emission and so on) of TPVTR dots in vitro, TPVTR dots were further introduced into long-term tumor tracking in vitro and in vivo. HepG2 cells incubated with TPVTR dots $\left(10 \mu \mathrm{g} \mathrm{mL}^{-1}\right)$ for $4 \mathrm{~h}$ were as the first generation cells. After the cells were cultured for a period of time, the fluorescent signals of cells were assessed by confocal fluorescence microscope and flow cytometry. Although the fluorescent intensity of cells gradually decreases, the cells still exhibit bright red fluorescence even up to 16 days (Figure 3A and Figure S6). The results of flow cytometry are consistent with confocal fluorescence microscope (Figure S7). All the results confirm that TPVTR dots can be utilized as a promising fluorescent probe for long-term tracking in vitro.

The experiment of TPVTR dots for long-term tracing in vivo was subsequently carried out. HepG2 cells $\left(5 \times 10^{6}\right.$ cells per mouse) labeled with TPVTR dots (10 $\mu \mathrm{g} \mathrm{mL}^{-1}$ ) were subcutaneously injected into flank of nude mice. After transplantion, a time-dependent optical imaging of a small animal in vivo was carried out. The transplanted site of tumor cells in mice emits bright red fluorescence (excitation around $510 \mathrm{~nm}$ and emission around 700 nm). After TPVTR dots-labeled HepG2 cells injected for $8 \mathrm{~d}$, the fluorescent signals still remain detectable (Figure 3B). Finally, the tumor-labled nude mice were euthanized. Then the tumor and other organs were analyzed by Bluck in vivo imaging system (Figure 3C). The fluorescent images of tumor and normal 
organs (e.g. heart, liver, spleen, lung and kidney) show that only the tumor displays the fluorescent signal. These results confirm the ability of TPVTR dots in long-term monitoring tumor growth in living organisms.

In order to further confirm that TPVTR dots have no side effect on the growth of nude mice, the nude mice were observed for 3 weeks after tail vein injection of $200 \mu \mathrm{L}$ of TPVTR dots solution $\left(20 \mu \mathrm{g} \mathrm{mL}^{-1}\right.$ in PBS solutions). The nude mice were euthanized and took out the major organs for H\&E staining (Figure S8). The major organs (such as lung, liver, heart, kidney, and spleen) show no significant apoptosis or necrosis, which further demonstrates that TPVTR dots exhibit little side effects. These results confirm that TPVTR dots are promising fluorescent nanomaterials for applications in vivo.

(A)

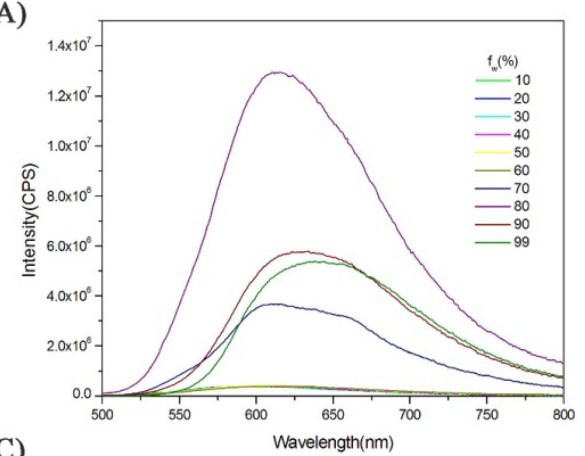

(C)

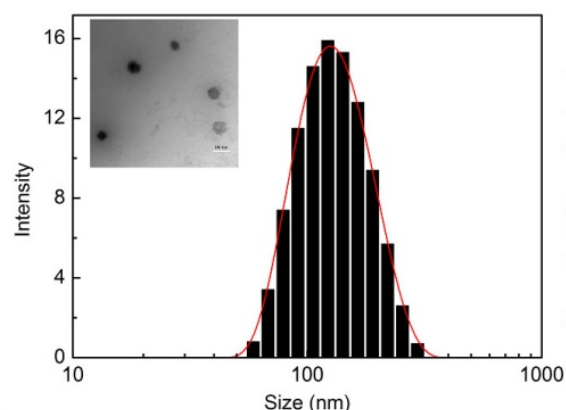

(E)

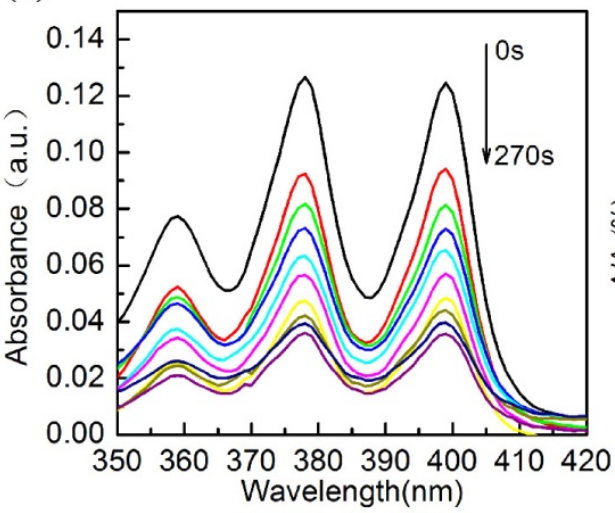

\section{Intracellular ROS Detection and In vivo Photodynamic Therapy}

To evaluate the ROS generation by TPVTR dots inside cells, a common oxidant-sensitive dye, DCFHDA (2,7-dichlorofluorescein diacetate, a cellpermeable green fluorescent probe) was selected to validate the intracellular ROS generation directly. Figure 4A shows that bright green fluorescence is observed in HepG2 cells under visible-light (400 750 $\mathrm{nm}, 100 \mathrm{~mW} \cdot \mathrm{cm}^{-2}$ ) irradiation after co-cultured with TPVTR dots, suggesting that intracellular ROS is generated. Meanwhile, HepG2 cells merely treated with light irradiation or TPVTR dots yield weak or even no fluorescence signal. These images prove that TPVTR dots in cells produce ROS under light irradiation, which has a great potential for photodynamic therapy.
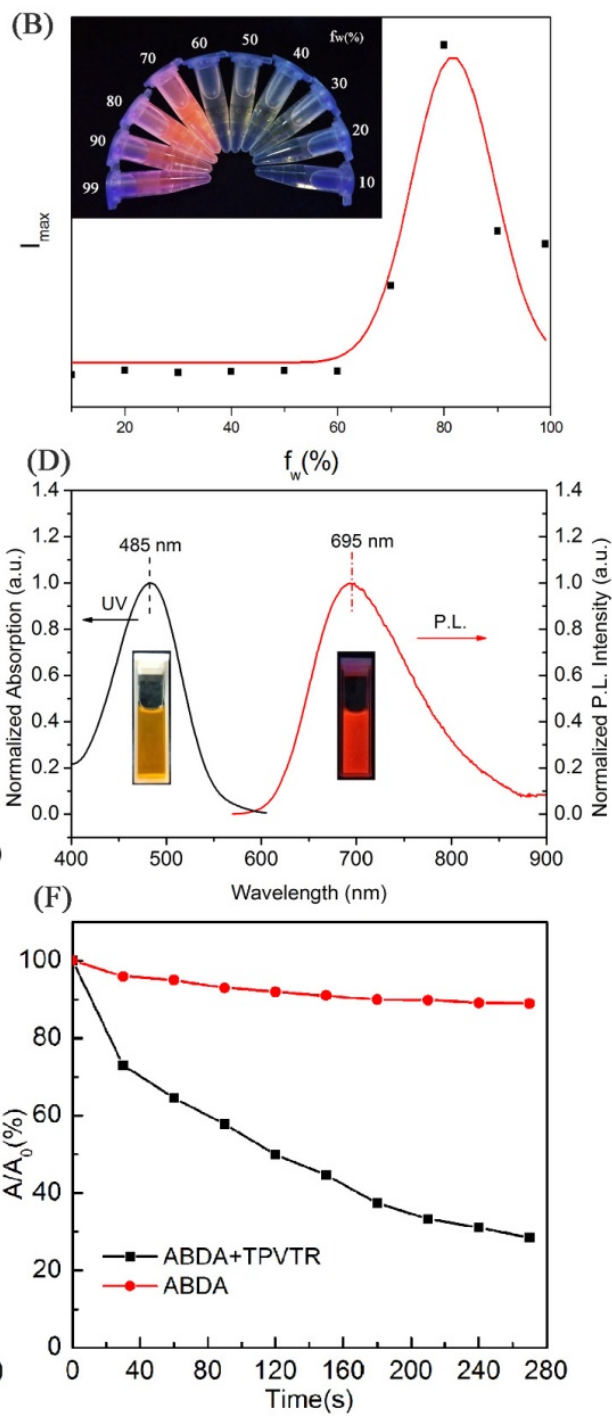

Figure 1. (A) PL spectra of TPVTR in water/THF mixture. (B) Trend diagram of fluorescent peaks versus the contents of water in water/THF mixture, Picture insert of (B): PL image of TPVTR in water/THF mixture. (C) Particle-size distribution of TPVTR dots in water studied via DLS experiment, picture insert of (C): TEM image of TPVTR dots, scale bar: $100 \mathrm{~nm}$. (D) Normalized UV and FL spectra of TPVTR dots in water. (E) Decomposition of ABDA by TPVTR dots under light irradiation $\left(400 \sim 750 \mathrm{~nm}, 200 \mathrm{~mW} \mathrm{~cm}{ }^{-2}\right)$ from $0 \mathrm{~s}$ to $270 \mathrm{~s}$. (F) ABDA decomposition rate by TPVTR dots under light irradiation, Ao is the absorbance of ABDA before white light irradiation, $A$ is the absorbance of ABDA after light irradiation for designated time. 
Meanwhile, photodynamic therapy efficacy of TPVTR dots toward HepG2 cells in vitro was further investigated by CCK-8 assays (Figure 4B). The cells viabilities remain almost $80 \%$ even treated with 50 $\mu \mathrm{g} \mathrm{mL} \mathrm{m}^{-1}$ of TPVTR dots, demonstrating low cytotoxicity in dark and good biocompatibility of TPVTR dots in living cells. By contrast, the viability of HepG2 cells dramatically decrease below $50 \%$ after treated with 25 $\mu \mathrm{g} \mathrm{mL}^{-1}$ of TPVTR dots under light illumination (100 $\mathrm{mW} \cdot \mathrm{cm}^{-2}, 10 \mathrm{~min}$ ), indicating the effective PDT efficiency.

At last, the in vivo photodynamic therapy effect was evaluated by tracing tumor ablation. The HepG2 cells $\left(5 \times 10^{6}\right.$ cells per mouse) were subcutaneously implanted into nude mice. Then the nude mice were randomly divided into four groups and treated with either injection of saline, light irradiation (400 750 $\mathrm{nm}, 100 \mathrm{~mW} \cdot \mathrm{cm}^{-2}$ ), intratumoral injection of TPVTR dots or light irradiation $\left(400 \sim 750 \mathrm{~nm}, 100 \mathrm{~mW} \cdot \mathrm{cm}^{-2}\right)$ after intratumoral injection of TPVTR dots while the volume of tumors reached approximately 100 200 $\mathrm{mm}^{3}$. The body weight and tumor size of nude mice were recorded every two days to evaluate the PDT efficiency in vivo up to 22 days. In order to avoid damage to the skin of mice, the irradiation of tumor site in nude mice was suspended for one minute after every irradiation. In addition, the temperature of tumor site before and after light irradiation (400 750 $\mathrm{nm}, 10 \mathrm{~min}$ ) are all below $42^{\circ} \mathrm{C}$, the results show that the temperature difference is only $1.67 \mathrm{C}$. As illustrated in Figure 5, the tumor of mice in injection

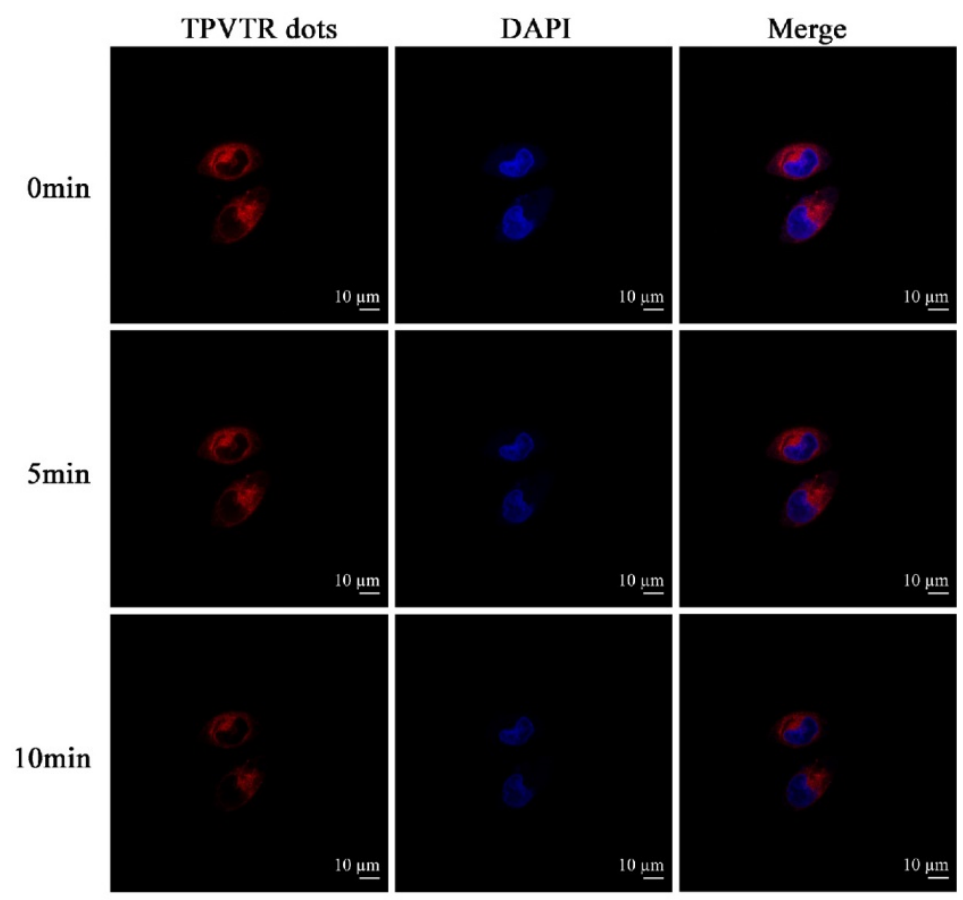

Figure 2. Photostability of TPVTR dots in HepG2 cells under continuous laser irradiation. of saline group grew rapidly, and there was no obvious difference with other control groups. In a marked contrast, the tumor growth in PDT group is significantly ablated after 21 days. In addition, there was no distinct difference in body weight between the all groups.The marked reduced tumor size by TPVTR dots results confirmed their effective PDT efficiency in vivo.

\section{Conclusions}

In summary, an effective NIR emissive organic fluorophore, TPVTR, with AIE characteristic has been designed and synthesized. The corresponding organic fluorescent nanoparticles, TPVTR dots, were fabricated and enjoyed NIR emission around $695 \mathrm{~nm}$ with a large Stokes shift $(210 \mathrm{~nm})$, excellent photostability, and good biocompatibility, which is suitable for long-term cell tracing in vitro and in vivo. Moreover, TPVTR dots offer good PDT effect in vitro and in vivo due to the high ROS generation efficiency, revealing that TPVTR dots are promising in image-guide therapy. This research highlights the efficient strategy for developing promising near-infrared organic fluorescent nanoparticles in advancing the field of bioimaging and further image-guide clinical applications.

\section{Methods}

\section{Materials}

All reagent and solvents were commercially available and used directly without further purification unless specified. Bromotriphenylethylene and 5-formylthiophene-2-boronic acid were purchased from Tokyo Chemical Industry (Japan). Cell Counting Kit-8 was purchased from Dojindo Laboratories (Kumamoto, Japan). DAPI, 4\% paraformaldehyde and Trypsin-EDTA Solution were purchased from Solarbio Co. Ltd. (China). DCFH-DA (2, 7-dichlorofluorescein diacetate) Detection Kit were purchased from Beyotime Biotechnology Co. Ltd. (China). Hematoxylin and Eosin Staining Kit were purchased from KeyGen Biotech. Co. Ltd. China).

\section{Characterization}

NMR spectra were measured via a Bruker $400 \mathrm{MHz}$ NMR with DMSO- $d_{6}$ or $\mathrm{CDCl}_{3}$. Infrared spectroscopy (IR) was performed with a Shimadzu FTIR-8100 spectrophotometer. High-resolution mass spectra was conducted on a Bruker Autoflex instrument. UV absorption spectra were recorded on Thermofisher Evolution 300 spectropolarimeter. FL spectra 
were obtained using a Thermofisher Lumina spectrofluorometer.

\section{Synthesis of TPVT-CHO}

The $\mathrm{PdCl}_{2}\left(\mathrm{PPh}_{3}\right)_{2}(262 \mathrm{mg}, 0.375 \mathrm{mmol}), 2 \mathrm{M}$ $\mathrm{Cs}_{2} \mathrm{CO}_{3}$ solution $(25 \mathrm{~mL})$, bromotriphenylethylene (5.03 g, $15 \mathrm{mmol})$, 5-formylthiophen-2-ylboronic acid $(4.68 \mathrm{~g}, 30 \mathrm{mmol})$, distilled toluene $(75 \mathrm{ml})$ and $\mathrm{EtOH}$ $(50 \mathrm{ml})$ were mixed in a $200 \mathrm{~mL}$ flask. After degassed by bubbling $\mathrm{N}_{2}$ for $1 \mathrm{~h}$, the reaction was carried out at $80^{\circ} \mathrm{C}$ for $24 \mathrm{~h}$. Then, the reaction mixture was poured into ice water, extracted with DCM for three times. The solvent was evaporated under vacuum and the resulting residue was purified by silica gel column chromatography using n-Hexane/Ethylacetate (4:1; $\mathrm{v} / \mathrm{v}$ ) for elution, affording TPVT-CHO as a yellow solid with $76 \%$ yield. ${ }^{1} \mathrm{H}$ NMR $\left(400 \mathrm{MHz}, \mathrm{DMSO}-d_{6}\right)$, $\delta$ (ppm): 9.85 9.86(s, $\mathrm{CHO}, 1 \mathrm{H}), 7.52 \sim 7.83(\mathrm{~m}, \mathrm{Ar}$, $13 \mathrm{H}), 7.43 \sim 7.47(\mathrm{~m}, \mathrm{Ar}, 3 \mathrm{H}), 7.12 \sim 7.14(\mathrm{~d}, \mathrm{Ar}, 1 \mathrm{H}) .{ }^{13} \mathrm{C}$ NMR (150 MHz, DMSO- $\left.d_{6}\right), \delta$ (ppm): 178.84, 157.66, 153.88, 144.53, 144.50, 144.48, 137.41, 137.11, 130.81, $130.44,128.55,128.37,128.05127 .33,126.73,119.88$,
$115.87,112.16$.

\section{Synthesis of TPVTR}

Rhodanine [42] (1.21 g, $5 \mathrm{mmol}), \mathrm{TPVT}-\mathrm{CHO}$ (1.83 $\mathrm{g}$, $5 \mathrm{mmol}$ ), and $\mathrm{NH}_{4} \mathrm{Ac}(500 \mathrm{mg}$ ) were added in HOAc $(20 \mathrm{ml})$ under $\mathrm{N}_{2}$ atmosphere. The resulting mixture was stirred at $120 \mathrm{C}$ for $12 \mathrm{~h}$. After cooling to room temperature, the precipitate was collected and washed with cold $\mathrm{MeOH}$. The crude product was recrystallized in $\mathrm{DCM} / \mathrm{EtOH}(1 / 4, \mathrm{v} / \mathrm{v})$ to obtain TPVTR as red solid. Yield: $2.25 \mathrm{~g}(74 \%) .{ }^{1} \mathrm{H}$ NMR (400 $\left.\mathrm{MHz}, \mathrm{CDCl}_{3}\right), \delta$ (ppm): 7.59 7.66(m, 3H), 7.44 7.47 $(\mathrm{m}, 3 \mathrm{H}), 7.36 \sim 7.37(\mathrm{~m}, 1 \mathrm{H}), 7.34 \sim 7.35(\mathrm{~m}, 1 \mathrm{H}), 7.30 \sim 7.32$ $(\mathrm{m}, 2 \mathrm{H}), 7.24 \sim 7.27(\mathrm{~m}, 5 \mathrm{H}), 7.19 \sim 7.20(\mathrm{~d}, 2 \mathrm{H}), 7.10 \sim 7.11$ $(\mathrm{m}, 3 \mathrm{H}), 7.01 \sim 7.04(\mathrm{~m}, 2 \mathrm{H}), 6.70 \sim 6.71(\mathrm{~d}, 1 \mathrm{H}) .{ }^{13} \mathrm{C} \mathrm{NMR}$ (100 MHz, $\left.\mathrm{CDCl}_{3}\right), \delta$ (ppm): 155.75, 144.86, 141.99, 141.90, 141.35, 137.22, 134.48, 132.80, 131.77, 131.69, 131.21, 130.57, 130.11, 129.41, 129.19, 128.64, 128.18, 127.64, 127.61, 127.05, 113.21, 122.98. IR ( $\left.\mathrm{v}^{-1}, \mathrm{KBr}\right)$, 3355, 3024, 2217, 1719, 1645, 1586, 1537, 1459, 1387, 1212, 1152, 1074, 757, 691. HRMS (ESI): $[\mathrm{M}+\mathrm{H}]^{+}$calcd for $\mathrm{C}_{37} \mathrm{H}_{23} \mathrm{~N}_{3} \mathrm{OS}_{2}$, found, 590.1345 .

(A)
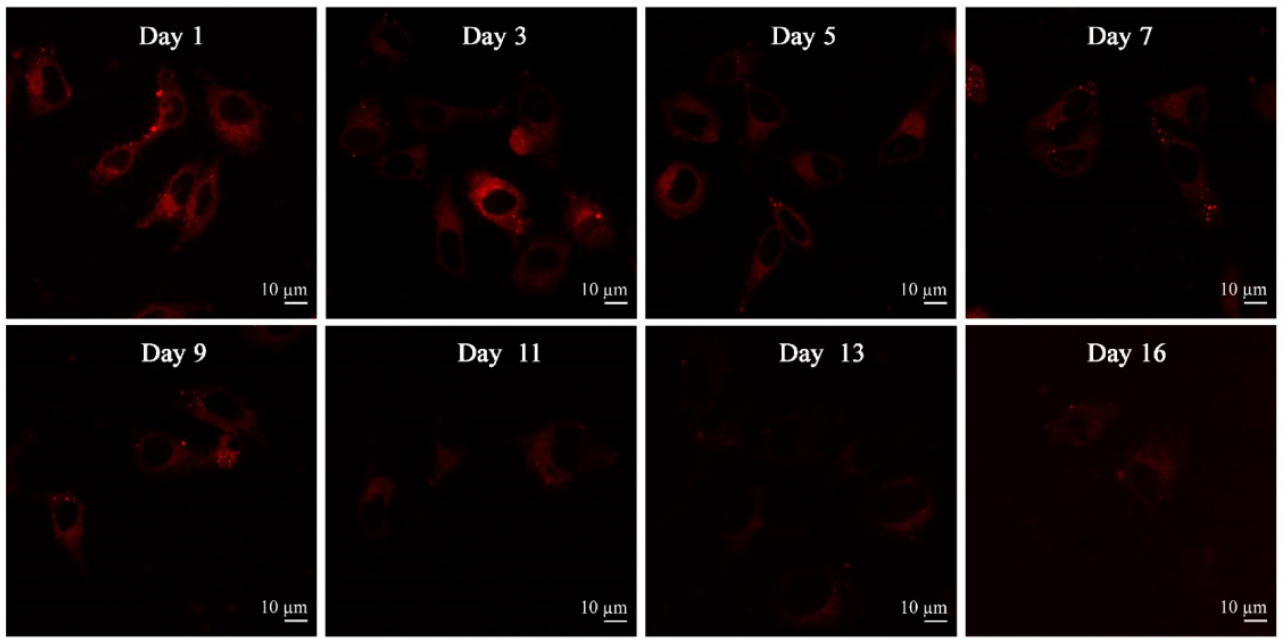

(B)
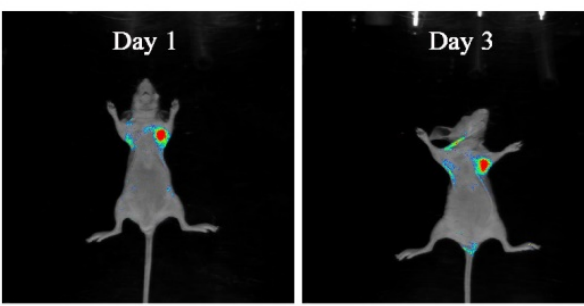

(C)
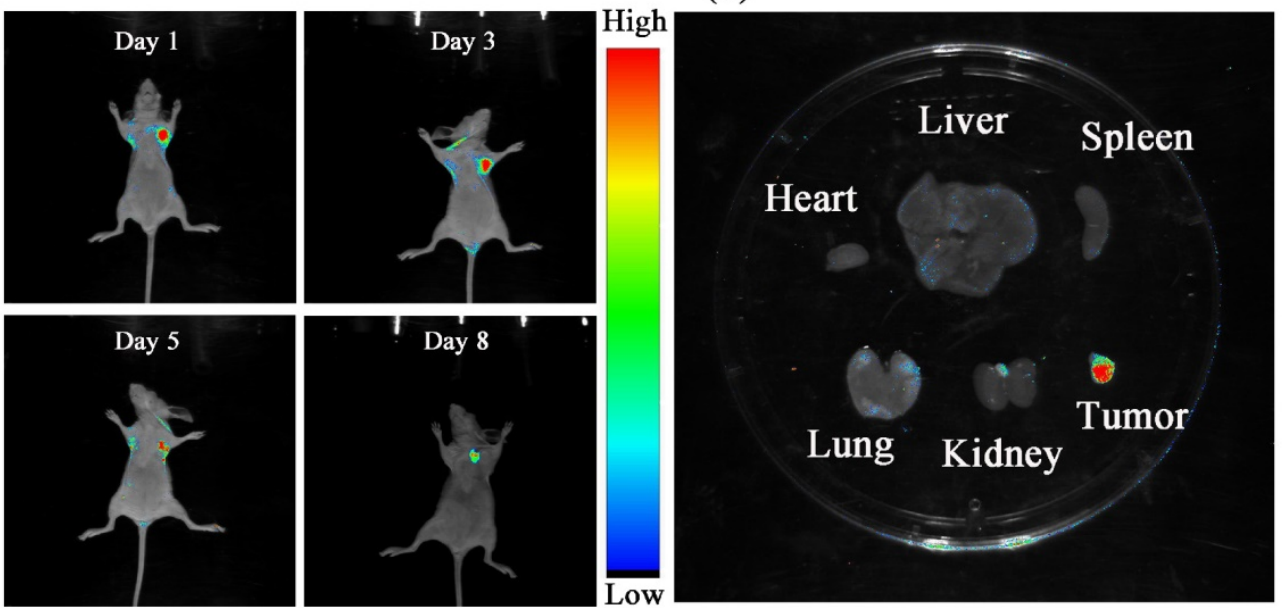

Figure 3. (A) CLSM images TPVTR dots-stained HepG2 cells at different time. (B) In vivo fluorescent imaging of tumor labeled by TPVTR dots. (C) Representative ex vivo fluorescent images of the isolated organs from the nude mice after 8 days tumor labeling 


\section{TPVTR dots fabrication}

TPVTR dots were fabricated via nanoprecipitation method by using Pluronic F-127 as the encapsulation. Generally, Pluronic F-127 (200 mg) and TPVTR $(20 \mathrm{mg})$ were added into THF $(2 \mathrm{~mL})$. After mixing for $0.5 \mathrm{~h}$, THF solution was drop added into the DI water $(10 \mathrm{~mL})$ under ultrasonic condition. The mixture was bubbling $\mathrm{N}_{2}$ for $2 \mathrm{~h}$ to completely remove the THF. After that, the free TPVTR was completed removed by dialysis to obtain the TPVTR dots.

\section{Extracellular ROS detection}

The extracellular ROS production of TPVTR upon irradiation was determined using a commercial ${ }^{1} \mathrm{O}_{2}$ probe, 9,10-anthracenediyl-bis(methylene) dimalonic acid (ABDA), as the indicator. A DMSO solution of TPVTR $(1 \mathrm{mM}, 5 \mu \mathrm{L})$ and a DMSO solution of ABDA $(10 \mathrm{mM}, 5 \mu \mathrm{L})$ were added into $990 \mu \mathrm{L}$ water. Then the mixed solution was exposed to white light $(400 \sim 750 \mathrm{~nm})$ from $0 \mathrm{~s}$ to $270 \mathrm{~s}$. The descending ABDA absorbance detected by ultraviolet spectrophotometer indicates the extracellular ROS production of TPVTR. As the control group, the DMSO solution of TPVTR (1 $\mathrm{mM}, 5 \mu \mathrm{L}$ ) was replaced by $5 \mu \mathrm{L}$ pure DMSO. The UV absorption of ABDA was detected after the same irradiation conditions.

\section{Cell culture}

TPVTR dots was diluted with culture medium to obtain a working solution $\left(10 \mu \mathrm{g} \mathrm{mL}^{-1}\right)$ for the subsequent cellular experiments. HepG2 cells were gifted from Nanfang Hospital (Guangzhou, China), and cultured in DMEM culture medium in a $5 \% \mathrm{CO}_{2}$ incubator at $37^{\circ} \mathrm{C}$.

\section{Photostability study}

To investigate the photostability of TPVTR dots, the parameters and procedures were as follows: HepG2 cells co-stained by TPVTR dots and DAPI were analyzed every 1 min under continuous laser irradiation. The fluorescent intensity of each image was evaluated. The photostability of TPVTR dots was assessed by the ratio between the fluorescent intensity at different time intervals during continuous laser irradiation and the initial value as a function of the exposure time.

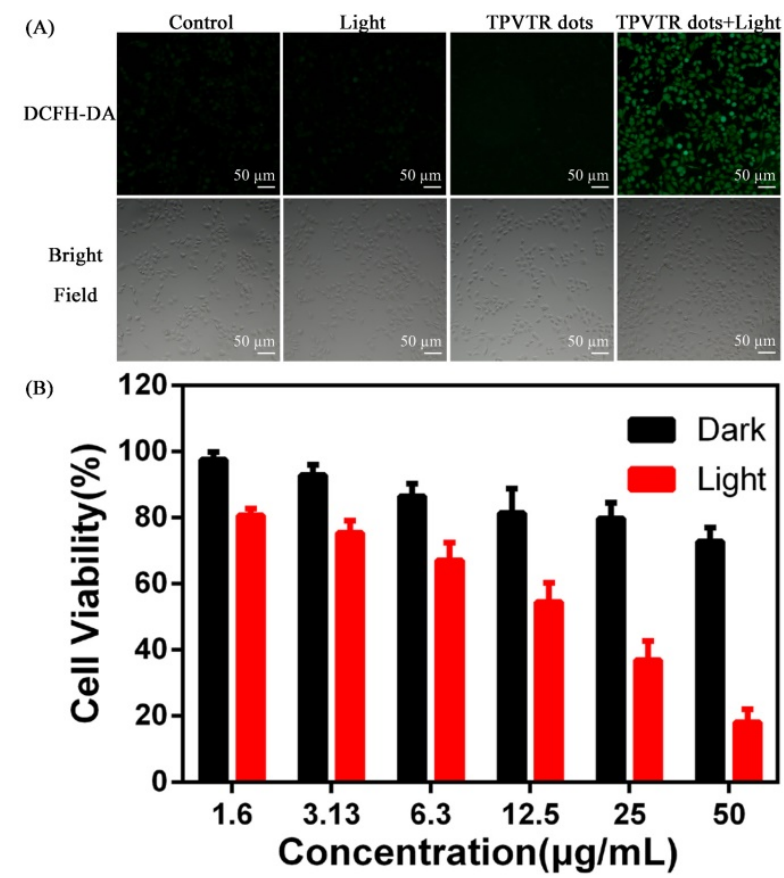

Figure 4. (A) Intracellular ROS detection using DCFH-DA as the sensor. (B) In vitro cytotoxicity of TPVTR dots without or with light irradiation (100 $\mathrm{mW} \cdot \mathrm{cm}^{-2}, 10 \mathrm{~min}$ )
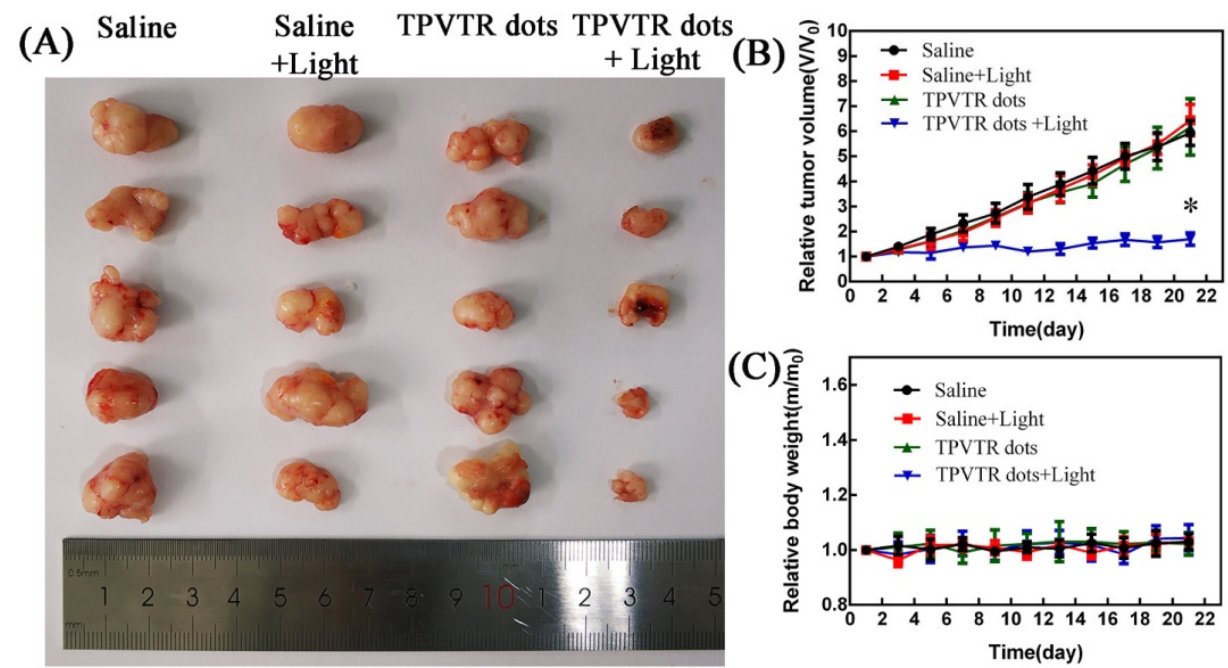

Figure 5. (A) Photographs of tumors on day 21 following indicated treatments. (B) Changes in tumor volumes in living mice following treatment with either saline, saline with light, TPVTR dots, TPVTR dots with light during the course of treatments (21 days), values were mean \pm SD ( $n=5$, *P < 0.05 ); (C) Body weight changes of the four groups during the course of treatments ( 21 days), $n=5$, data are the mean \pm SD 


\section{Long-term cell tracking in vitro}

HepG2 cells were seeded in confocal microscope dish with $5 \times 10^{4}$ cells. After attachment, the old medium was replaced by TPVTR dots $\left(10 \mu \mathrm{g} \mathrm{mL}^{-1}\right)$. After incubation at $37 \mathrm{C}$ for $4 \mathrm{~h}$, the cells were washed with PBS buffer, detached by EDTA-trypsin and resuspended in culture medium. Upon dilution, the cells were subcultured in wells containing cell culture coverslips for different cell generations. At specified time intervals, the cells were trypsinized, suspended and fixed with $4 \%$ paraformaldehyde for $15 \mathrm{~min}$. The stained cells were imaged using a confocal fluorescence microscope (LSM 880 with Airyscan, Carl Zeiss). On the other hand, after treatment with TPVTR dots for $4 \mathrm{~h}$, the cells at the corresponding time interval were wished, digested and collected for flow cytometry analysis using BD LSRFortessa for quantitative study of fluorescent intensities. A batch of blank cells without any treatment was used as the control group.

\section{Intracellular ROS detection}

The intracellular ROS production of TPVTR dots was determined using DCFH-DA (2,7-dichlorofluorescein diacetate) Detection Kit. HepG2 cells were seeded in 6-well plate at $37 \mathrm{C}$ in humidified environment containing $5 \% \quad \mathrm{CO}_{2}$. After overnight culturing, the cells were treated with PBS or TPVTR dots $\left(10 \mu \mathrm{g} \mathrm{mL}^{-1}\right)$ for $4 \mathrm{~h}$, respectively. Then, the cells were washed with PBS, and incubated with $10 \mu \mathrm{M}$ DCFH-DA in serum-free DMEM medium for $20 \mathrm{~min}$ at $37 \mathrm{C}$ in humidified environment containing $5 \%$ $\mathrm{CO}_{2}$. The cells were washed with PBS again, and irradiated with white light $(400 \sim 750 \mathrm{~nm})$ for $4 \mathrm{~min}$. After irradiation, the cells were imaged using an inverted fluorescence microscope (Axio Observer, Carl Zeiss) (excited at $488 \mathrm{~nm}$, monitored at $525 \mathrm{~nm}$ ).

\section{In vitro cytotoxicity}

The cells were seeded in 96 well plates and incubated overnight at $37 \mathrm{C}$ in humidified environment containing $5 \% \mathrm{CO}_{2}$. Subsequently, the cells were treated with TPVTR dots in $100 \mathrm{~mL}$ of fresh culture medium at various concentrations (from 1.6 50 $\mu \mathrm{g} / \mathrm{mL}$ ) and subjected to the dark or light irradiation $\left(400 \sim 750 \mathrm{~nm}, 100 \mathrm{~mW} \mathrm{~cm}^{-2}\right)$ for $10 \mathrm{~min}$. After treatment, the cells were returned to the incubator for another $24 \mathrm{~h}$. The culture medium was removed, and $100 \mu \mathrm{L}$ of $10 \mu \mathrm{M}$ CCK-8 was added into each well and incubated for $4 \mathrm{~h}$. The absorbance at $450 \mathrm{~nm}$ of each well was measured using a BIOTEK ELX80 enzyme-linked immunosorbent assay reader. The relative cell viability was calculated as follows: cell viability $(\%)=\mathrm{OD}($ sample $) \times 100 / \mathrm{OD}($ control $)$, in which OD (control) was obtained when without samples and OD (sample) was obtained with the addition of TPVTR dots at various concentration.

\section{In vivo long-term monitoring tumor}

The in vivo experiment was conducted on the adult BALB/c nude mice (15 16 g). All animal experiments were approved by the local Animal Ethics Committee of Southern Medical University, China. After incubation with $20 \mu \mathrm{g} \mathrm{mL}-1$ TPVTR for $4 \mathrm{~h}$ at $37 \mathrm{C}$, HepG2 cells $\left(5 \times 10^{6}\right.$ cells in $0.1 \mathrm{~mL}$ DMEM) were subcutaneously injected into the nude mice. Fluorescent imaging were recorded using an IVISH Spectrum Imaging System after 1, 3, 5, and 8 days. After 8 days, tumor and other organs including heart, liver, spleen, lung, kidney were excised for ex vivo fluorescence imaging.

\section{Phototherapeutic efficacy of TPVTR dots in vivo}

HepG2 liver cancer cells $\left(5 \times 10^{6}\right.$ cells per mouse) were subcutaneously injected into the nude mice. When the tumor volumes reached 100 $200 \mathrm{~mm}^{3}$, the mice were randomly assigned to 4 groups and treated with different treatments every four days: 1) saline, 2) saline with light irradiation, 3) TPVTR dots, 4) TPVTR dots with light irradiation. The mice in groups 3 and 4 were intratumoral injected with TPVTR dots at the equivalent dose of $10 \mathrm{mg} / \mathrm{kg}$. After injection, the light irradiation $\left(400 \sim 750 \mathrm{~nm}, 100 \mathrm{~mW} \cdot \mathrm{cm}^{-2}, 10 \mathrm{~min}\right)$ was administrated one minute after every one minute irradiation. The body weight and tumor size of nude mice were recorded with electronic balance and vernier calipers every two days.

\section{Hematoxylin and eosin (H\&E) staining analysis}

After injected TPVTR dots solution into the tail vein of nude mice at the equivalent dose of 10 $\mathrm{mg} / \mathrm{kg}$, the physical conditions of the nude mice were monitored and observed, including their general status, e.g., skin and activities for 16 days. Subsequently, the mice were sacrificed and then the liver, spleen, lung, kidney and heart were collected for H\&E staining. The major organs were fixed in formalin and cryosectioned at $10 \mathrm{~mm}$ thickness onto slides and stained with H\&E (KeyGen Biotech. Co. Ltd., Nanjing, China) according to the manufacturer's instructions.

\section{Acknowledgements}

This work was financially supported by the National Natural Science Foundation of China (No. 81671749) and the Natural Science Foundation of Guangdong Province, China (No. 2015A030313205). 


\section{Supplementary Material}

Supplementary figures.

http://www.ntno.org/v03p0156s1.pdf

\section{Competing Interests}

The authors have declared that no competing interest exists.

\section{References}

1. Shen Y, Shuhendler AJ, Ye D, Xu JJ, Chen HY. Two-photon excitation nanoparticles for photodynamic therapy. Chem Soc Rev. 2016; 45: 6725-6741.

2. Kamkaew A, Lim SH, Lee HB, Kiew LV, Chung LY, Burgess K. BODIPY dyes in photodynamic therapy. Chem Soc Rev. 2013; 42: 77-88.

3. Feng G, Wu W, Xu S, Liu B. Far red/near infrared AIE dots for image-guided photodynamic cancer cell ablation. ACS Appl Mater Interfaces. 2016; 8:21193-21200.

4. Ge J, Lan M, Zhou B, Liu W, Guo L, Wang H, Jia Q, Niu G, Huang X, Zhou $\mathrm{H}$. A graphene quantum dot photodynamic therapy agent with high singlet oxygen generation. Nature Commun. 2014; 5: 4596.

5. Celli JP, Spring BQ, Imran R, Evans CL, Samkoe KS, Sarika V, Pogue BW, Tayyaba H. Imaging and photodynamic therapy: mechanisms, monitoring, and optimization. Chem Rev. 2010; 110: 2795-2838.

6. Zhou Z, Song J, Nie L, Chen X. Reactive oxygen species generating systems meeting challenges of photodynamic cancer therapy. Chem Soc Rev. 2016; 45: 6597-6626.

7. Lucky SS, Soo KC, Zhang Y. Nanoparticles in photodynamic therapy. Chem Rev. 2015; 115: 1990-2042.

8. Agostinis P, Berg K, Cengel KA, Foster TH, Girotti AW, Gollnick SO, Hahn SM, Hamblin MR, Juzeniene A, Kessel D. Photodynamic therapy of cancer: an update. CA Cancer J Clin. 2011; 61: 250-281.

9. Liu LH, Qiu WX, Zhang YH, Li B, Zhang C, Gao F, Zhang L, Zhang XZ. A Charge Reversible Self-Delivery Chimeric Peptide with Cell Membrane-Targeting Properties for Enhanced Photodynamic Therapy. Adv Funct Mater. 2017; 27: 1700220.

10. Wang H, Chao Y, Liu J, Zhu W, Wang G, Xu L, Liu Z. Photosensitizer-crosslinked in-situ polymerization on catalase for tumor hypoxia modulation \& enhanced photodynamic therapy. Biomaterials. 2018; 181: 310-317.

11. Zhang $\mathrm{C}$, Chen WH, Liu LH, Qiu WX, Yu WY, Zhang XZ. An $\mathrm{O}_{2}$ Self-Supplementing and Reactive-Oxygen-Species-Circulating Amplified Nanoplatform via $\mathrm{H}_{2} \mathrm{O} / \mathrm{H}_{2} \mathrm{O}_{2}$ Splitting for Tumor Imaging and Photodynamic Therapy. Adv Funct Mater. 2017; 27: 1700626.

12. Li F, Du Y, Liu J, Sun H, Wang J, Li R, Kim D, Hyeon T, Ling D. Responsive Assembly of Upconversion Nanoparticles for $\mathrm{pH}$-Activated and Near-Infrared-Triggered Photodynamic Therapy of Deep Tumors. Adv Mater. 2018; 30: 1802808

13. Ye S, Rao J, Qiu S, Zhao J, He H, Yan Z, Yang T, Deng Y, Ke H, Yang H, Zhao Y, Guo Z, Chen H. Rational Design of Conjugated Photosensitizers with Controllable Photoconversion for Dually Cooperative Phototherapy. Adv Mater. 2018; 30: 1801216.

14. Turan IS, Yildiz D, Turksoy A, Gunaydin G, Akkaya EU. A Bifunctional Photosensitizer for Enhanced Fractional Photodynamic Therapy: Singlet Oxygen Generation in the Presence and Absence of Light. Angew Chem Int Ed. 2016; 55: 2875-2878.

15. Sun $\mathrm{Y}, \mathrm{Hu} \mathrm{H}$, Zhao N, Xia T, Yu B, Shen C, Xu FJ. Multifunctional polycationic photosensitizer conjugates with rich hydroxyl groups for versatile water-soluble photodynamic therapy nanoplatforms. Biomaterials. 2017; 117: 77-91.

16. Li X, Lee D, Huang JD, Yoon J. Phthalocyanine-Assembled Nanodots as Photosensitizers for Highly Efficient Type I Photoreactions in Photodynamic Therapy. Angew Chem Int Ed. 2018; 57: 9885-9890.

17. Wang D, Niu L, Qiao ZY, Cheng DB, Wang J, Zhong Y, Bai F, Wang H, Fan H. Synthesis of Self-Assembled Porphyrin Nanoparticle Photosensitizers. ACS Nano. 2018; 12: 3796-3803.

18. Yu J, Zhang X, Hao X, Zhang X, Zhou M, Lee CS, Chen X. Near-infrared fluorescence imaging using organic dye nanoparticles. Biomaterials. 2014 ; 35 : 3356-3364

19. Xu R, Huang L, Wei W, Chen $X$, Zhang $X$, Zhang X. Real-time imaging and tracking of ultrastable organic dye nanoparticles in living cells. Biomaterials. 2016; 93: 38-47.

20. Ni JS, Zhang P, Jiang T, Chen Y, Su H, Wang D, Yu ZQ, Kwok RTK, Zhao Z, Lam JWY, Tang BZ. Red/NIR-Emissive Benzo[d]imidazole-Cored
AIEgens: Facile Molecular Design for Wavelength Extending and In Vivo Tumor Metabolic Imaging. Adv. Mater. 2018; 30: 1805220.

21. Guo B, Sheng ZH, Hu DH, Liu CB. Zheng HR, Liu B. Through Scalp and Skull NIR-II Photothermal Therapy of Deep Orthotopic Brain Tumors with Precise Photoacoustic Imaging Guidance. Adv Mater. 2018; 30: 1802591.

22. Jin G, He R, Liu Q, Lin M, Dong Y, Li K, Tang BZ, Liu B, Xu F. Near-infrared light-regulated cancer theranostic nanoplatform based on aggregation-induced emission luminogen encapsulated upconversion nanoparticles. Theranostics. 2019; 9: 246-264.

23. Zheng $X$, Ge J, Wu J, Liu W, Guo L, Jia Q, Ding $Y$, Zhang $H$, Wang P. Biodegradable hypocrellin derivative nanovesicle as a near-infrared light-driven theranostic for dually photoactive cancer imaging and therapy. Biomaterials. 2018; 185: 133-141.

24. Yan X, Niu G, Lin J, Jin AJ, Hu H, Tang Y, Zhang Y, Wu A, Lu J, Zhang S, Huang $\mathrm{P}$, Shen B, Chen $X$. Enhanced fluorescence imaging guided photodynamic therapy of sinoporphyrin sodium loaded graphene oxide. Biomaterials. 2015; 42: 94-102.

25. Gu X, Zhang X, Ma H, Jia S, Zhang P, Zhao Y, Liu Q, Wang J, Zheng X, Lam JWY, Ding D, Tang BZ. Corannulene-Incorporated AIE Nanodots with Highly Suppressed Nonradiative Decay for Boosted Cancer Phototheranostics In Vivo. Adv Mater. 2018; 30: 1801065.

26. Li J, Meng X, Deng J, Lu D, Zhang X, Chen Y, Zhu J, Fan A, Ding D, Kong D, Wang Z, Zhao Y. Multifunctional Micelles Dually Responsive to Hypoxia and Singlet Oxygen: Enhanced Photodynamic Therapy via Interactively Triggered Photosensitizer Delivery. ACS Appl Mater Interfaces. 2018; 10: 17117-17128.

27. Li W, Zheng C, Pan Z, Chen C, Hu D, Gao G, Kang S, Cui H, Gong P, Cai L. Smart hyaluronidase-actived theranostic micelles for dual-modal imaging guided photodynamic therapy. Biomaterials. 2016; 101: 10-19.

28. Li J, Lee WY, Wu T, Xu J, Zhang K, Hong WD, Li R, Li G, Bian L. Near-infrared light-triggered release of small molecules for controlled differentiation and long-term tracking of stem cells in vivo using upconversion nanoparticles. Biomaterials. 2016; 110: 1-10.

29. Li H, Wang $\mathrm{P}$, Deng $\mathrm{Y}$, Zeng $\mathrm{M}$, Tang $\mathrm{Y}$, Zhu WH, Cheng $\mathrm{Y}$. Combination of active targeting, enzyme-triggered release and fluorescent dye into gold nanoclusters for endomicroscopy-guided photothermal/photodynamic therapy to pancreatic ductal adenocarcinoma. Biomaterials. 2017; 139: 30-38.

30. Xuan M, Shao J, Zhao J, Li Q, Dai L, Li J. Magnetic Mesoporous Silica Nanoparticles Cloaked by Red Blood Cell Membranes: Applications in Cancer Therapy. Angew Chem Int Ed. 2018; 57: 6049-6053.

31. Feng X, Yan W, Chen S, Guo W, Liu D, Yang X, Wang S, Liang XJ, Zhang $\mathrm{J}$. Biomimetic $\mathrm{O}_{2}$-Evolving metal-organic framework nanoplatform for highly efficient photodynamic therapy against hypoxic tumor. Biomaterials. 2018; 178: 83-94.

32. Hu F, Mao D, Kenry, Wang Y, Wu W, Zhao D, Kong D, Liu B. Metal-Organic Framework as a Simple and General Inert Nanocarrier for Photosensitizers to Implement Activatable Photodynamic Therapy. Adv Funct Mater. 2018; 28: 1707519

33. Gu X, Kwok RTK, Lam JWY, Tang BZ. AIEgens for biological process monitoring and disease theranostics. Biomaterials. 2017; 146: 115-135.

34. Yan L, Zhang Y, Xu B, Tian W. Fluorescent nanoparticles based on AIE fluorogens for bioimaging. Nanoscale. 2016; 8: 2471-2487

35. Xia Q, Chen Z, Yu Z, Wang L, Qu J, Liu R. Aggregation-Induced Emission-Active Near-Infrared Fluorescent Organic Nanoparticles for Noninvasive Long-Term Monitoring of Tumor Growth. ACS Appl Mater Interfaces. 2018; 10: 17081-17088.

36. Oian $Y$, Wang $Y$, Jia F, Wang Z, Yue C, Zhang W, Hu Z, Wang W. Tumor-microenvironment controlled nanomicelles with AIE property for boosting cancer therapy and apoptosis monitoring. Biomaterials. 2019; 188: 96-106.

37. Yuan Y, Zhang CJ, Kwok RTK, Xu S, Zhang R, Wu J, Tang BZ, Liu B. Light-Up Probe for Targeted and Activatable Photodynamic Therapy with Real-Time In Situ Reporting of Sensitizer Activation and Therapeutic Responses. Adv Funct Mater. 2015; 25: 6586-6595.

38. Yuan Y, Zhang CI, Gao M, Zhang R, Tang BZ, Liu B. Specific light-up bioprobe with aggregation-induced emission and activatable photoactivity for the targeted and image-guided photodynamic ablation of cancer cells. Angew Chem Int Ed. 2015; 54: 1780-1786.

39. $\mathrm{Hu} \mathrm{F}, \mathrm{Xu} \mathrm{S}$, Liu B. Photosensitizers with Aggregation-Induced Emission: Materials and Biomedical Applications. Adv Mater. 2018; 30: 1801350.

40. Wang D, Su H, Kwok RTK, Shan G, Leung ACS, Lee MMS, Sung HHY, Williams ID, Lam JWY, Tang BZ. Facile Synthesis of Red/NIR AIE Luminogens with Simple Structures, Bright Emissions, and High Photostabilities, and Their Applications for Specific Imaging of Lipid Droplets and Image-Guided Photodynamic Therapy. Adv Funct Mater. 2017; 7: 1704039 . 
41. Wu W, Mao D, Hu F, Xu S, Chen C, Zhang CJ, Cheng X, Yuan Y, Ding D, Kong D, Liu B. A Highly Efficient and Photostable Photosensitizer with Near-Infrared Aggregation-Induced Emission for Image-Guided Photodynamic Anticancer Therapy. Adv Mater. 2017; 29: 1700548.

42. Zhang Y, He L, Wu J, Wang K, Wang J, Dai W, Yuan A, Wu J, Hu Y. Switchable PDT for reducing skin photosensitization by a NIR dye inducing self-assembled and photo-disassembled nanoparticles. Biomaterials. 2016; 107: 23-32.

43. Cai X, Mao D, Wang C, Kong D, Cheng X, Liu B. Multifunctional Liposome: A Bright AIEgen-Lipid Conjugate with Strong Photosensitization. Angew Chem Int Ed. 2018; 57: 16396-16400.

44. Wang D, Lee MMS, Shan G, Kwok RTK, Lam JWY, Su H, Cai Y, Tang BZ. Highly Efficient Photosensitizers with Far-Red/Near-Infrared Aggregation-Induced Emission for In Vitro and In Vivo Cancer Theranostics. Adv Mater. 2018; 30: 1802105. 